Ecological parasitology

Ecological Aspects of Parasitology. Edited by C. R. Kennedy. Pp. x+474. (North-Holland: Amsterdam and Oxford, 1976.) Dfl 145; $\$ 57.95$.

Parasitism is an ecological term, and parasitology is essentially an ecological study. Some aspects of parasite ecology have received extensive coverage for many years, especially those related to qualitative and quantitative epideniology, where the unit of study is the infection rather than the parasite.

The stated object of this book is to remedy the situation whereby some aspects have received little attention. The aspects chosen in the first section concern the parasite outside the host: dispersal (C. R. Kennedy), host location (A. J. MacInnis) and host selection, (J. C. Holmes); entry into the host (D. W. T. Crompton), feeding (C. Arme), interaction between parasites (D. Halvörsen), and host responses to parasitic infection (D. Wakelin). The bulk of the book is in part two. Here, "Habitat" is seen as the immediate surroundings of the parasite. There are two chapters on fish: skin (G. C. Kearn) and gills (C. H. Fernando and C. Hanek); and the remaining chapters deal with the special features of parasitic life in different mammalian or avian organs and cells. In the final chapter, R. M. Anderson discusses the interaction of host and parasite populations.

With 24 authors covering so wide a field, consistency and uniformity are not to be expected or even desired. If the intellectual verbosity of chapter one were maintained throughout, the book would be hard indeed to read. Particularly noteworthy is yet another definition of parasitism, first credited with elegant beauty, then, mercifully, discredited as being possibly applicable to all organisms!

The rest of the book is clear and stimulating. The only truly unique feature of the parasitic habitat is the specific antagonistic reaction that it produces. This aspect is given extensive treatment. D. R. Arthur describes how amputee mice, unable to groom, are subject to massive infestations of lice; D Wakelin gives a lucid comparison between the immune processes of invertebrates and vertebrates, and A. D. Befus and R. B. Podesta describe the special immunological features of the intestine.

Most other aspects of parasite ecology have clear parallels in free living organisms. Useful comparisons are made but unfortunately not ex-

panded with both sessile and planktonic marine animals. More such comparisons could have been enlightening.

The great value of this book lies in the descriptions of various organs from a parasitological viewpoint. Almost every author points out areas of extensive ignorance in his field, and it is clear that too few physiologists, biochemists and physicians have a clear idea of what ecology is about. The book will surely be useful as a

\section{Electron theory of small molecules}

Introduction to the Electron Theory ot Small Molecules. By A. C. Hurley. Pp. 329. £12; \$26.25. Electron Correlation in Small Molecules. By A. C. Hurley. Pp. 276. £10.50; \$23. (Academic: London and New York, 1977.)

THESE two books are perhaps meant to be read together. The author states in his preface to the Introduction that the book consists of the "earlier and simpler chapters of what was originally conceived of as a comprehensive onevolume work". As such, the Introduction is to provide what the author calls a "royal road from basic quantum mechanics to the various theories which are now yielding such detailed information on molecular interactions", some of which are discussed in the Electron Correlation volume.

The Introduction provides an account of the basic theory of potential energy curves and surfaces, an outline of the variational method for constructing approximate electronic wave functions and a short account of the (generalised) virial and HellmannFeynmann theorems. It also provides a brief, but self-contained account of molecular symmetry and quite extended accounts of the determinantal approach and of molecular orbitals and the Hartree-Fock method. The accounts are presented chiefly in the context of results for diatomic molecules (although small polyatomics occasionally receive a passing mention) and a chapter each is devoted to detailed consideration of the hydrogen ion and of the hydrogen molecule.

The book is regrettably something of a disappointment to the reviewer, if for no other reason than that it seems so old-fashioned. A suspicion of this may be gleaned from the first few pages in which it becomes clear that the author is going to use c.g.s. units; but it is re-inforced, for example, by his treatment of spin-coupling and of methods for solving the SCF equations, stimulant for discussion and further investigation. It is a little disappointing that in so extensive a series of reviews there is a sad lack of coordinating thought. Perhaps, though, that is what the book will help to produce.

R. W. Ashford

R. W. Ashford is Senior Lecturer in the Department of Pathology, University of Papua, New Guinea, on secondment from the Liverpool School of Tropical Medicine.

for which he gives no references more recent than 1967. Indeed, if one looks through the text one gains the impression that the phrase "recent work" should really be synonymous with "ten-year-old work". This criticism should not, of course, be construed as a call for "trendiness", but there is work in the past five years in the areas quoted above (and indeed in other areas in the book) to which the attention of the reader should be called even in an introductory book like this.

As one would expect from an author of Dr Hurley's distinction, there are a lot of good things in the Introduction. There is, for example, a splendid and precise account of Koopmans' theorem, a very nice heuristic account of the various cusp conditions in the wave function, some very helpful material on the continuous groups in the section on symmetry, a brief and clear account of the RKR method, and many more. There are, however, some funny slips. The author says, for example, that in the quadratic approximation to the potential, the equation for nuclear vibrational motion in the diatomic is the simple harmonic oscillator equation. But the student who has read his Eyring, Walter and Kimball Quantum Chemistry knows that this is just not true. The equation (2.60) from which he begins his account of the generalised HellmanFeynmann theorem seems to be simply wrong at an elementary level (see the author's own original paper, Proc. $R$. Soc. A226, 170 or Appendix 1 in Pople et al. Jl Chem. Phys. 49, 2960; 1968 , and in view of Dr Hurley's contributions to the foundations of this part of the subject this (if the reviewer is not mistaken) is quite incredible. Be that as it may, the discussion in this part of the book has nothing like the subtlety or power of the discussion of the same material offered by Epstein in his recent book (The Variation Method in Quantum Chemistry, Academic: London, 1974); and Epstein's book is not referred to at all.

At a somewhat different level, the author's discussion of natural orbitals

(continued on p654) 